\title{
3D Gait Abnormality Detection Employing Contactless IR-UWB Sensing Phenomenon
}

\author{
Soumya Prakash Rana*, Member, IEEE, Maitreyee Dey, Member, IEEE, Mohammad Ghavami, Senior \\ Member, IEEE, and Sandra Dudley, Member, IEEE
}

\begin{abstract}
Gait disorder diagnosis and rehabilitation is one area where human perception and observation are highly integrated. Predominantly, gait evaluation, comprises technological devices for gait analysis such as, dedicated force sensors, cameras, and wearable sensor based solutions, however they are limited by insufficient gait parameter recognition, post processing, installation costs, mobility, and skin irritation issues. Thus, the proposed study concentrates on the creation of a widely deployable, noncontact and non-intrusive gait recognition method from impulse radio ultra wideband (IR-UWB) sensing phenomenon, where a standalone IR-UWB system can detect gait problems with less human intervention. A 3D human motion model for gait identification from IR-UWB has been proposed with embracing spherical trigonometry and vector algebra to determine knee angles. Subsequently, normal and abnormal walking subjects were involved in this study. Abnormal gait subjects belong to the spastic gait category only. The prototype has been tested in both the anechoic and multipath environments. The outcomes have been corroborated with a simultaneously deployed Kinect Xbox sensor and supported by statistical graphical approach Bland and Altman (B\&A) analysis.
\end{abstract}

Index Terms-Gait, Impulse Radio Ultra Wideband (IR-UWB), 3D Gait Identification, Kinect Xbox Sensor, Bland and Altman Plot.

\section{INTRODUCTION}

G AIT or human locomotion is a bipedal, sinusoidal, forward propulsive movement of the human body where, upper and lower limbs coordinate simultaneously. This is a complex translatory motion including the brain, spinal cord, peripheral nerves, muscles, bones and joints [1]. Physically, each and every bone of the human skeleton participates in the process but, empirically the bones of the pelvis and lower limb are considered to realise this repetitive motion. There has been a growing interest to better characterise gait or detect abnormalities when people are unable to walk in a normal way, particularly among practitioners, physiotherapists, biomedical engineers, neurologists and rehabilitation societies. Abnormalities occur because of injuries to the legs or feet, arthritis, infections in the soft tissue of the legs, broken bones in feet and legs, birth defects, infections in the inner ear, cerebral palsy, stroke, tendonitis, neuropathy, conversion disorder or other psychological disorders and shin splints [2]. These directly affect the flexion and extension, abduction and adduction, internal and external rotation of legs, hip joint, knee joint, ankle, toes, hindfoot, and forefoot [1]. Abnormal gait is categorised as one of five types based on the symptoms or

All author are members of the Centre for BioEngineering, London South Bank University, 103 Borough Road, London SE1 0AA, United Kingdom appearance of an individual's walk; spastic gait, scissors gait, steppage gait, waddling gait, and propulsive gait [2].

Traditionally, there are two ways employed to assess gait: qualitative and quantitative. Patients (or, subjects) are requested to walk to measure retrospective gait events and are observed under clinical conditions by doctors or experts in qualitative gait assessment, whereas, a number of technological devices are employed for objective measurement to estimate gait parameters in a quantitative gait assessment. The devices can be broadly classified into three categories; nonwearable sensors (NWS), wearable sensors (WS), and hybrid systems (HS) [3]. NWS systems include image processing, employing fixed sensors placed on the ground surrounded by cameras for data collection. Ugbolue et. al., proposed an augmented video based portable system (AVPS) for clinical gait analysis where the gait data are collected using a light indicator and video cameras to extract the kinematic event and tibia inclination angle [4]. Pfister et. al., captured 3D gait characteristics in the sagittal plane through a Kinect sensor. They analysed hip and knee angular displacement during flexion and extension of muscles, and the work was validated through the Vicon Nexus model [5]. Corazza et. al., developed another form of NWS system, a markerless motion capture (MMC) system where ten joint centers and the body shape are learned through linear regression (LR) to recognise gait kinematic and human body morphology [6]. Force platforms are also well known for gait assessment in controlled laboratory and observation settings. Lim et. al., prototyped a force platform to measure step phases through ground reaction forces (GRF) acquired from sensors employed for inspecting lower limb exoskeleton [7]. Impulse radio ultrawide band (IR-UWB) pulsed Doppler radar is also in NWS category utilised by researchers because of it's high bandwidth and precise performance in describing human motion [8], [9]. Koo et al. proposed UWB based system to differentiate human gait from vital signs by analysing spectograms. This explored range and time-frequency analysis associated with human locomotion [10]. Seifert et al. implemented IR-UWB microDoppler ( $\mu$-D) signature model to analyse gait using timefrequency variation obtained from wavelet transform (WT). Subsequently, coefficients are fed into a classification method to recognise abnormal gait patterns for rehabilitation and assisted living purposes [11]. Mokhtari et al. prototyped a UWB model to identify gait signatures of different person's employing region of interest (ROI) approach. Further, backscattered energies are measured from the ROI and used as features to categorise different persons using support vector 
machine (SVM), random forest (RF), logistic regression (LR), $k$-nearest neighbour $(k \mathrm{NN})$ and neural network $(\mathrm{NN})$ models [12]. Potluri et. al. measured the significant gait parameters using wearable plantar pressure and inertial sensors to investigate differences between normal and abnormal walking patterns identified by a long short-term memory (LSTM) model to predict the risk of fall [13]. Similar research by Gao et. al. employed inertial sensors to obtain abnormal gait pattern step information, including hemiplegic, tiptoe, and cross-threshold gait. The step features were employed in a hybrid model of LSTM and convolutional neural network (CNN) to classify abnormal gait [14]. Ren et al. modelled a system to extract $\mu$-D features from UWB radar for gait stride identification employing short time state-space method (STSSM) [15]. In addition, Orovic et al. proposed a human gait classification method which relies on motion signatures from arm and leg movements obtained through a continuous wave (CW) radar signal [16]. Widely used WSs are force sensors (FS), accelerometers, gyroscope, extensometers, goniometers, electromyography, and active markers etc. placed at the hips, feet, etc. to assess gait characteristic [17]. Bruening and Ridge reviewed existing algorithms related to gait events, velocity, and acceleration of foot/heel strike, toe off. The data are collected through foot and sacrum markers initially to make ground truth information and the simulation repeated to obtain said gait parameters. The work claims that the existing study carried out by Ghoussayni et. al. [18] to measure sagittal velocity is reliable for clinical gait application while a single algorithm is not sufficient to measure all gait events [19]. Greene et. al., developed an adaptive algorithm to measure initial contact (IC) and terminal contact (TC) timing of the foot via gyroscopes. Additionally, it calculates angular velocity, stride length, swing time, stance time, step times from human gait. The study includes force measurement plates and an optical motion capture system to compare obtained results with the proposed algorithm [20]. Bugane et. al., prototyped an accelerometer based gait characterisation model to measure single and double support interval from gait event. Also, it determines spatial and temporal parameters such as, stride length and duration, cadence, and speed. Popular gait analysis methods, stereophotogrametry and dynamometry are employed for validation [21]. Industry is working in parallel to academic research towards the creation of robust and efficient gait analysis tools. CONTEMPLAS produced a professional motion analysis software TEMPLO for clinical gait analysis [22]. This is a hybrid approach for gait measurement solution that uses both NWS and WS systems for diagnosis. Tekscan makes pressure mapping, force measurement, and tactile sensors for clinical gait assessment [23]. They primarily focus on force platforms, but use additional instruments such as, video scan and electromyography (EMG) analysis to construct a hybrid tools for gait characterisation.

The NWSs (such as, force platform, pressure plate, GRF sensor, etc.) for gait analysis are parameter specific e.g., a force platform only delivers step duration and step length which are not sufficient to detect abnormal gait. The clinical setup needs several devices to take decisions on gait patterns which increases time, space, and cost complexity. Subsequently, cameras such as, laser range scanners, infrared sensor camera, time of flight (ToF) camera are also a type of NWSs for gait characterisation but consume huge effort to differentiate between foreground (patient or object) and background (stationary backdrop) before any calculation of significant biomechanical parameters. The WSs such as, active markers, force sensors, magnetometers, etc. measure gait parameters more precisely compared to NWSs as they measures biomechanical parameters of gait trajectory in 3D which describes walk are better than the parameters obtained from NWS. Although, the patients do feel uncomfortable when several markers are placed on the body during examination. The field needs alteration methods preferably NWS without camera integration which would be adequate to measure human gait in non-contact and non-invasive manner providing precise decisions in gait characterisation. One potential technology is UWB Doppler radar which had been employed in continuous mode and operated in frequency modulation. But, the $\mathrm{CW}$ UWB radar does not provide propagation delay information for each pulse to measure frequency shift which restricts it only to calculate rate of change of range. A handful of UWB Doppler based gait identification works are accomplished using impulse radio but focus on spectogram and $\mu$-D which can provide the frequency of lower movement and walking speed respectively. However, the prospects of IR-UWB has not been fully explored which could provide 3D gait quantification and have advantages such as, mobility, ease of installation, remote access, cost efficiency over typical NWS and WS.

\section{A. Contribution}

This study develops an IR-UWB pulsed Doppler radar based prototype for quantitative human gait analysis demonstrating a non-contact and non-intrusive 3D human motion model. The initial model previously proposed by the authors in [24] is now augmented and improved to determine further significant gait parameters. The contributions this work are: (i) The radar pulse energy flow has been transformed into a wave vector using spherical trigonometry and vector algebra to capture the back scattered pulses from human motion in 3D. Here, anatomical planes such as, frontal, sagittal, and transverse plane are elucidated by motion width, range, and height of movement respectively to represent the unit vectors. (ii) The study has created a mathematical model to express the right and left knee angles as cosine angles that can be computed by the product of the Euclidean magnitudes of the two vectors formed, through motion width, range, and height. (iii) The real data collection process has been extended involving a greater number of human participants (twenty four hitherto) following ethical approval guidance. The developed prototype has been tested in both an anechoic chamber and multi-path environment employing real data for normal and abnormal walking to distinguish walking patterns. (iv) Simultaneously, the Kinect Xbox One sensor has been chosen to corroborate the obtained results from the proposed IR-UWB prototype, where the Kinect's skeleton joints have been symbolised as vectors and projected as one vector onto another to derive the right and left knee angles. $(v)$ The Blant and Altman (B\&A) 
plot has been considered to compare the two fold experimental results obtained from both IR-UWB and Kinect to establish an agreement between them. The proposed IR-UWB is found to be superior or equivalent to Kinect in both the environments from this research study.

The remaining sections of the article are organised as follows; a description of the laboratory set-up, UWB data acquisition, proposed methodology, Kinect sensor and data interpretation procedure, B\&A analysis is discussed in Section III, experimental results have been demonstrated in Section III. Section IV concludes the paper and provides future research directions.

\section{METhODS}

The knee angle has a compelling effect on gait and is the angle between the straight line joining the lateral malleolus fibula head and a straight line joining the lateral femoral epicondyle great trochanter. The knee angle is a pivotal parameter for gait biomechanics and it shows significant variation during abnormal walking. The participants involved in this study have the common problem of spasticity for the muscles around the knee during leg swing, but also display a lower level of spasticity around the ankle and hip joints. Thus, the proposed prototype described here focuses on the knee angle (right and left) calculation of human gait during flexion and the extension of the leg muscles. A flow chart of this work is provided in Figure 1. The work is divided into seven parts; laboratory setup, participant recruitment and their subjective data collection, data processing, proposed spherical trigonometric approach and its vectorisation for determining knee angles, Kinect Xbox sensor calibration and its vectorisation for determining knee angles, and the result comparison through a B\&A plot to justify the potentiality of the proposed work. Each of these aforementioned tasks have been detailed in the following sections.

\section{A. Laboratory Set-up}

A Time Domain PulsON P410 monostatic radar module (P410 MRM) have been used to collect all the physiological UWB sensing phenomenon reported here and published in [24], [25], [26], [27], [28], are shown in Figure 2a The device is a monostatic pulsed Doppler radio transceiver which utilises TW-TOF omni-directional range measurement techniques as a hybrid ranging radio and a radar sensor device for nonintrusive human gait measurement. The device has been configured before data collection and the same configuration has been maintained for both of the chosen (tested) environments. The PulsON P410 module generates Gaussian pulses and transmits first order derivative of the Gaussian pulse providing high power efficiency by delivering extremely low power spectral density (PSD) to mitigate the influence of surrounding multipath environments. In addition, the nanosecond duration Gaussian pulses have low duty cycle resulting in a high pulse repetition rate (PRR) of $10 \mathrm{MHz}$ enabling improved detection of human movement of transmitting the radio frequency (RF) range of a lower frequency limit $3.1 \mathrm{GHz}$ to an upper frequency limit $5.3 \mathrm{GHz}$, with the centre frequency at $4.3 \mathrm{GHz}$,

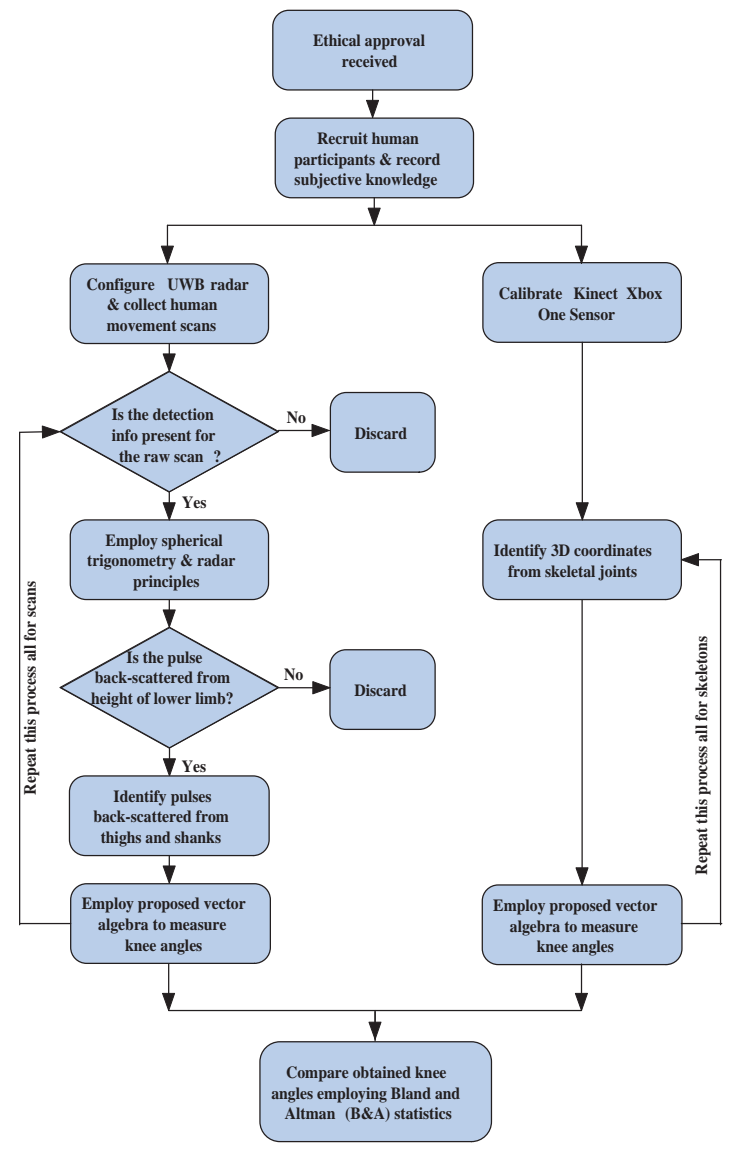

Fig. 1: The flow of processing UWB data and extracting gait information.

and a bandwidth of $2.2 \mathrm{GHz}$ (where the fractional bandwidth of P410 device is 51.16\%) addresses to FCC restrictions [29] for power. Transmission power to the antenna port is specified as $-12.64 \mathrm{dBm}$ for safe RF transmission [29]. The scan time window for this experiment is 87.84 nanoseconds (ns) long, but the first 5 ns of the radar signal is neglected in order to negate noise and direct path interference between transmitter and receiver antenna, thus the signal during the first $5 \mathrm{~ns}$ is filtered from subsequent analysis. The scan interval is set to $25000 \mu s$. The received reflected pulsed signals are sampled in 61 picosecond steps, which results in a sampling frequency $f_{s}=16.39 \mathrm{GHz}$, with a Pulse Repetition Interval (PRI) of approximately $100 \mathrm{~ns}$.

\section{B. Data Acquisition}

Twenty four human participants were involved in this data collection process. Initially, gender and anatomical information (height, length of the limbs) have been recorded for each individual, as shown in Figure $2 \mathrm{~d}$. The recorded information from all twenty four subjects is tabulated in Table I. where length of leg, length of thigh, and length of shank have been denoted by LoL, LoT, and LoS respectively. Full ethical approval (Reference Number: Eng_01Dec2017) was gained from London South Bank University, where the research code of practice and ethical guidelines are governed by the 
university ethics panel (UEP). All procedures performed in this study were in accordance with the ethical standards of the institutional and/or national research committee and with the 1964 Helsinki declaration and its later amendments or comparable ethical standards. Both anechoic chamber and multi-path/normal (laboratory) environments have been used for the data collection. These two environments are shown in Figure $2 \mathrm{~b}$ and $2 \mathrm{c}$.

TABLE I: Subjective data of twenty four individuals.

\begin{tabular}{|c|c|c|c|c|c|}
\hline No & Gender & Height $(\mathrm{m})$ & LoL $(\mathrm{m})$ & LoT $(\mathrm{m})$ & LoS $(\mathrm{m})$ \\
\hline 1 & Female & 1.58 & 0.85 & 0.43 & 0.42 \\
\hline 2 & Female & 1.54 & 0.83 & 0.42 & 0.41 \\
\hline 3 & Female & 1.64 & 0.88 & 0.45 & 0.43 \\
\hline 4 & Female & 1.73 & 0.93 & 0.47 & 0.46 \\
\hline 5 & Female & 1.62 & 0.87 & 0.44 & 0.43 \\
\hline 6 & Female & 1.71 & 0.91 & 0.46 & 0.45 \\
\hline 7 & Female & 1.69 & 0.91 & 0.46 & 0.44 \\
\hline 8 & Male & 1.67 & 0.88 & 0.45 & 0.43 \\
\hline 9 & Male & 1.76 & 0.91 & 0.46 & 0.45 \\
\hline 10 & Male & 1.71 & 0.88 & 0.45 & 0.43 \\
\hline 11 & Male & 1.72 & 0.88 & 0.45 & 0.43 \\
\hline 12 & Male & 1.64 & 0.84 & 0.42 & 0.42 \\
\hline 13 & Male & 1.78 & 0.92 & 0.47 & 0.45 \\
\hline 14 & Male & 1.79 & 0.92 & 0.46 & 0.46 \\
\hline 15 & Male & 1.78 & 0.92 & 0.47 & 0.45 \\
\hline 16 & Male & 1.78 & 1.00 & 0.52 & 0.48 \\
\hline 17 & Male & 1.75 & 1.02 & 0.52 & 0.50 \\
\hline 18 & Male & 1.72 & 1.00 & 0.51 & 0.49 \\
\hline 19 & Female & 1.55 & 0.95 & 0.50 & 0.45 \\
\hline 20 & Female & 1.53 & 0.94 & 0.50 & 0.44 \\
\hline 21 & Male & 1.76 & 1.03 & 0.54 & 0.49 \\
\hline 22 & Male & 1.78 & 1.01 & 0.53 & 0.48 \\
\hline 23 & Female & 1.65 & 0.97 & 0.50 & 0.47 \\
\hline 24 & Female & 1.58 & 0.95 & 0.49 & 0.46 \\
\hline
\end{tabular}

\section{Azimuth and Elevation Angles}

To assist in the differentiation of separate body areas, azimuth and elevation angles are considered. This is further explained in the Experimental section. Figure 3a shows the elevation and azimuth angle at a particular time, where $\triangle O A B, \triangle O A B^{\prime}, \triangle O C B$, and $\triangle O C B^{\prime}$ are drawn from the received pulsed radar signal. Here, $O$ is considered as the radar receiver, which is fixed at a point of height $O P$ from the ground. Therefore, $B C$ and $C B^{\prime}$ represent the height of a moving object from the radar line of sight (LOS) $O A$. The moving body section is elevated from the radar LOS at an angle $\theta$ and below the LOS at an angle $\theta^{\prime}$. Here, $\triangle O A B \cong \triangle O A B^{\prime}$ and $\triangle O C B \cong \triangle O C B^{\prime}$, therefore the height $B C$ and $C B^{\prime}$ can be determined from the trigonometric relationships. Only the calculation of $B C$ from $\triangle O A B$ is explained. Let, the angle between $B C$ and $O B$ be $\alpha$. The travelled distances are $O A, O B$, and $O B^{\prime}$ in propagation delays $t_{1}, t_{2}$ and $t_{2}^{\prime}$ by the pulses, where $t_{1}>t_{2}, t_{1}>t_{2}^{\prime}$ and $O A>O B, O A>O B^{\prime}$. Therefore, the change of distance is $(O A-O B)=\Delta d$, the change of time is $\left(t_{1}-t_{2}\right)=\Delta t$, and speed of light or pulse is $c$. Therefore, pulse can travel the distance in $\Delta t$ is $B C=\Delta t \times c$. From the trigonometric ratio in right triangle $\triangle O C B$,

$$
\begin{array}{r}
\cos \alpha=\frac{B C}{O B} \Rightarrow B C=O B \times \cos \alpha \\
\Rightarrow \alpha=\cos ^{-1}\left[\frac{\Delta t \times c}{O B}\right]
\end{array}
$$

Therefore, if the height of a moving object at a particular time is $h$ then,

$$
h=O B \times \cos \alpha
$$

This calculation has the same outcome when $t_{1}<t_{2}, t_{1}<$ $t_{2}^{\prime}$ and $O A<O B, O A<O B^{\prime}$.

Figure 3 a displays the calculation for the azimuth angle to determine the position or orientation of moving limbs toward the radar. The spherical system measures the azimuth angle in a counter clockwise direction from the exact north of the receiver end denoted by $\phi$. Let, the moving limb be deviated at an angle $\phi$, where the travelled distances are $X Y$ and $X W$ in propagation delay $t_{1}, t_{2}$. Thus, the change of distance is $(X Y-X W)=Y Z$ at the time interval $\left(t_{1}-t_{2}\right)=\Delta t$. The object is deviated from the exact north of the receiver. Now, $Y Z$ is approximately equivalent to the arc $Y W$ created by the object at angle $\phi$. Therefore, $\phi$ is calculated from the radian measure, and equivalent degree conversion is,

$$
\phi=\frac{Y Z \times 360^{\circ}}{X Y \times 2 \times \pi}
$$

Therefore, the position or the coordinate of a pulse hitting a human body would be found by considering range, elevation, and azimuth calculations. Let, a pulse which has backscattered from human body have its arc, range, height are $a$, $r, h$ respectively. Once, the position of back-scattered pulses from a human body has been identified, the points have been considered as vector (e.g., $a \hat{i}+r \hat{j}+h \hat{k}$ where, $\hat{i}, \hat{j}$, and $\hat{k}$ are unit vectors of three planes) in a $3 \mathrm{D}$ space to determine the gait parameters. The subscripts of $a, r$, and $h$ have been used throughout the article to denote arc, range, and height of a back-scattered pulse. The properties of vector algebra has been applied to measure the step length, hip angle, and knee joint angles for each participant using the a priori knowledge of human body sections and detailed in the following sections.

\section{Knee Angle Calculation from IR-UWB Model}

The human knee joint has two sections, thigh and shank. Thus, human gait creates an angle between these two muscles during the walking process. The angle increases during muscle extension (i.e., the straightening of the legs) and decreases during muscle flexion (i.e., articulation of legs). The change of knee joint angle is significant to characterize human gait. Figure $3 \mathrm{~b}$ shows a human walking posture where, two points $\overrightarrow{L_{T}}, \overrightarrow{L_{S}} \in \mathbb{R}^{3}$ Euclidean space at time $t$ have been assumed on thigh and shank of left leg respectively. The dot product of these two points provides the acute angle $\gamma_{L}$ between them whereas, the measurement of the obtuse angle $\left(\beta_{L}\right)$ is 


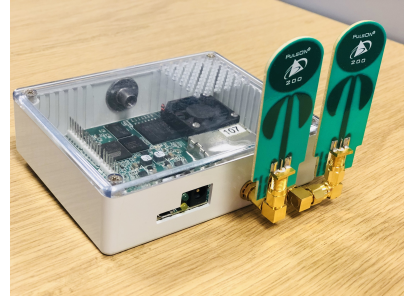

(a) UWB P410 radar module.

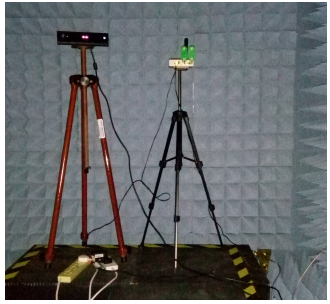

(b) Anechoic chamber.

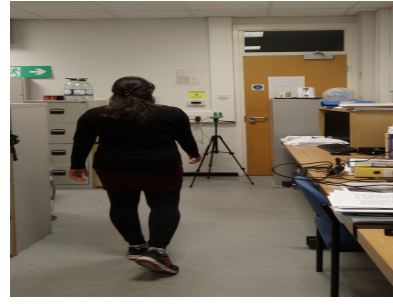

(c) Normal environment.

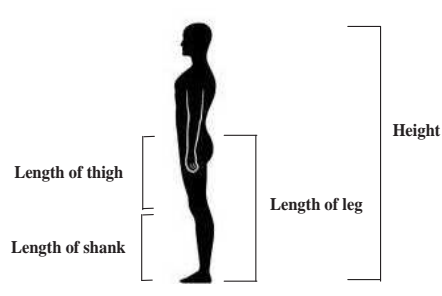

(d) Measurement of body parts.

Fig. 2: UWB device and the environments during data collection.

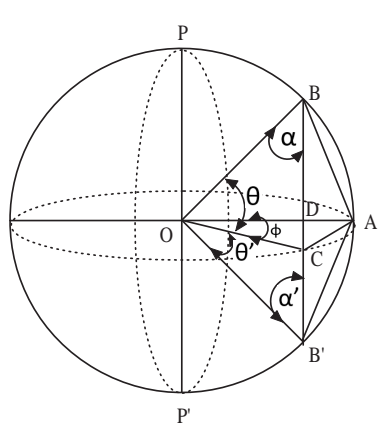

(a) Elevation and azimuth angle calculation.

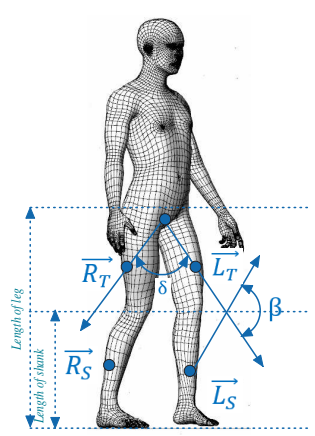

(b) Vectorization

Fig. 3: Three dimensional sphere consideration and vectorisation of back-scattered UWB pulse.

anatomically more significant. The detailed calculations of $\gamma$ and $\beta$ have been included in Eq. 4 and Eq. 5

$$
\begin{aligned}
\overrightarrow{L_{T}} \cdot \overrightarrow{L_{S}} & =\left|\overrightarrow{L_{T}}\right|\left|\overrightarrow{L_{S}}\right| \cos \gamma \\
\Rightarrow \cos \gamma_{L} & =\frac{\overrightarrow{L_{T}} \cdot \overrightarrow{L_{S}}}{\left|\overrightarrow{L_{T}}\right|\left|\overrightarrow{L_{S}}\right|} \\
\Rightarrow \cos \gamma_{L} & =\frac{\left(a_{1} \hat{i}+r_{1} \hat{j}+h_{1} \hat{k}\right) \cdot\left(a_{2} \hat{i}+r_{2} \hat{j}+h_{2} \hat{k}\right)}{\left|a_{1} \hat{i}+r_{1} \hat{j}+h_{1} \hat{k}\right|\left|a_{2} \hat{i}+r_{2} \hat{j}+h_{2} \hat{k}\right|} \\
\Rightarrow \cos \gamma_{L} & =\frac{\left(a_{1} a_{2}+r_{1} r_{2}+h_{1} h_{2}\right)}{\sqrt{a_{1}^{2}+r_{1}^{2}+h_{1}^{2}} \sqrt{a_{2}^{2}+r_{2}^{2}+h_{2}^{2}}} \\
\Rightarrow \quad \gamma_{L} & =\cos ^{-1}\left(\frac{\left(a_{1} a_{2}+r_{1} r_{2}+h_{1} h_{2}\right)}{\sqrt{a_{1}^{2}+r_{1}^{2}+h_{1}^{2}} \sqrt{a_{2}^{2}+r_{2}^{2}+h_{2}^{2}}}\right)
\end{aligned}
$$

Therefore, the obtuse knee angle $\left(\beta_{L}\right)$ for the left leg,

$$
\beta_{L}=180^{\circ}-\gamma_{L}
$$

Similarly, the acute knee angle $\gamma_{R}$ between $\overrightarrow{R_{T}}$ and $\overrightarrow{R_{S}}$ for right leg has been determined in Eq. 6.

$$
\gamma_{R}=\cos ^{-1}\left(\frac{\left(a_{3} a_{4}+r_{3} r_{4}+h_{3} h_{4}\right)}{\sqrt{a_{3}^{2}+r_{3}^{2}+h_{3}^{2}} \sqrt{a_{4}^{2}+r_{4}^{2}+h_{4}^{2}}}\right)
$$

Therefore, the obtuse knee angle for right leg has been included in Eq. 7.

$$
\beta_{R}=180^{\circ}-\gamma_{R}
$$

\section{E. Calibration of Kinect Xbox One}

The results obtained from the proposed model have been corroborated by measuring the knee angles with the Microsoft Kinect Xbox One. It includes a 3D image and voice sensor and employs time-of-flight (TOF) technology to deliver high resolution, low latency, light independent 3D image sensing. The Kinect sensor tracks 3D human skeleton using colour and depth sensors [30]. It has the potential as a low cost, accurate gait motion analysis tool which has good correlation with VICON Nexus motion capture system for hip, knee, and stride timing measurements. Furthermore, the study shows error is low and correlation high for stride and knee angle measurement compared to hip angular measurements carried out by Kinect and VICON sensor [31]. The proposed work aims to characterize human gait in a non-intrusive manner, so the device has been calibrated to obtain colour and skeleton only from the video. Frames per second (FPS) has been fixed at 30 for colour and depth sensor for video acquisition. The camera has a field view of $70^{\circ}$ horizontal and $60^{\circ}$ vertical. The camera sensor operates at range from 0.8 to 4.2 meters in one room only (unlike the model developed which has through wall capability) from the device. It tracks the skeleton from a moving body posture (as shown in Figure 4) and provides 3D joint coordinates. The Kinect sensor delivers 20 skeletal data (3D joint coordinates) at standing condition from the body posture. This skeletonization process is similar to the proposed prototype permitting the validation of the work via the Kinect sensor. Figure $4 \mathrm{~b}$ shows the 20 joints (white markers) from a human body where, the validation process has used only 6 joints from lower limb of a human body such as, the hip left $(\overrightarrow{H L})$, knee left $(\overrightarrow{K L})$, ankle left $(\overrightarrow{A L})$, hip right $(\overrightarrow{H R})$, knee right $(\overrightarrow{K R})$, and ankle right $(\overrightarrow{A R})$. Then the vector algebra has been employed on these joints to validate the proposed outcomes such as, step size, number of steps, speed, hip angle, and knee angles (for both left and right leg). Let, the vectors $\overrightarrow{H L}, \overrightarrow{K L}, \overrightarrow{A L}, \overrightarrow{H R}, \overrightarrow{K R}, \overrightarrow{A R} \in \mathbb{R}^{n}$ in Euclidean $n$-space. The component form of these vectors have been denoted as, $\overrightarrow{H L}=a_{5} \hat{i}+r_{5} \hat{j}+h_{5} \hat{k}, \overrightarrow{K L}=a_{6} \hat{i}+r_{6} \hat{j}+h_{6} \hat{k}, \overrightarrow{A L}=$ $a_{7} \hat{i}+r_{7} \hat{j}+h_{7} \hat{k}, \overrightarrow{H R}=a_{8} \hat{i}+r_{8} \hat{j}+h_{8} \hat{k}, \overrightarrow{K R}=a_{9} \hat{i}+r_{9} \hat{j}+h_{9} \hat{k}$, and $\overrightarrow{A R}=a_{10} \hat{i}+r_{10} \hat{j}+h_{10} \hat{k}$ where, subscripts with $a, r, h$ represents the distance from $\hat{i}, \hat{j}, \hat{k}$ planes respectively. These vectors have been further used to determine parameters for gait characterization in the following sections. 


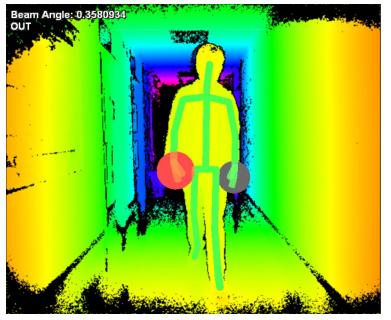

(a) Frame from color sensor.

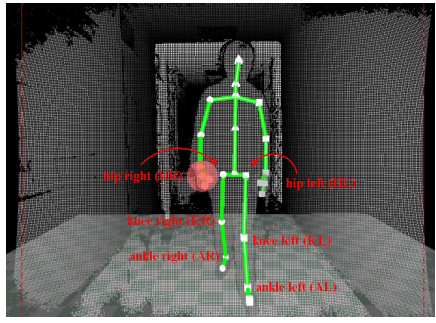

(b) Frame from depth sensor.
Fig. 4: Sample video frame of human gait tracked through Kinect color and depth sensor.

\section{F. Knee Angle Calculation from Kinect}

The knee angles (left and right) have been measured in a similar way to the hip joint calculation using the vector dot products. The knee and ankle joints (shown in Figure $4 \mathrm{~b}$ ) from skeletal data of both legs have been used to calculate knee angles of human gait. In the case of the left leg, the connecting line between vectors $\overrightarrow{H L}$ and $\overrightarrow{K L}$ would be spanned through the vector $\overrightarrow{L_{T k}}=a_{56} \hat{i}+r_{56} \hat{j}+h_{56} \hat{k}$ where $a_{56}=\left(a_{5}-\right.$ $\left.a_{6}\right), r_{56}=\left(r_{5}-r_{6}\right), h_{56}=\left(h_{5}-h_{6}\right)$ and the straight line between points $\overrightarrow{K L}$ and $\overrightarrow{A L}$ would span through the vector $\overrightarrow{L_{S k}}=a_{67} \hat{i}+r_{67} \hat{j}+h_{67} \hat{k}$ where $a_{67}=\left(a_{6}-a_{7}\right), r_{67}=$ $\left(r_{6}-r_{7}\right), h_{67}=\left(h_{6}-h_{7}\right)$. The dot product of $\overrightarrow{L_{T k}}$ and $\overrightarrow{L_{S k}}$ provides the acute angle between these two whereas, inner knee angle would be the obtuse angle between them. The acute angle has been denoted by $\gamma^{\prime}{ }_{L}$ and detailed in Eq. 8 .

$$
\begin{aligned}
\overrightarrow{L_{T k}} \cdot \overrightarrow{L_{S k}} & =\left|\overrightarrow{L_{T k}}\right|\left|\overrightarrow{L_{S k}}\right| \cos \gamma^{\prime} \\
\Rightarrow \cos \gamma^{\prime}{ }_{L} & =\frac{\overrightarrow{L_{T k}} \cdot \overrightarrow{L_{S k}}}{\left|\overrightarrow{L_{T k}}\right|\left|\overrightarrow{L_{S k}}\right|} \\
\Rightarrow \cos \gamma^{\prime}{ }_{L} & =\frac{\left(a_{56} \hat{i}+r_{56} \hat{j}+h_{56} \hat{k}\right) \cdot\left(a_{67} \hat{i}+r_{67} \hat{j}+h_{67} \hat{k}\right)}{\left|a_{56} \hat{i}+r_{56} \hat{j}+h_{56} \hat{k}\right|\left|a_{67} \hat{i}+r_{67} \hat{j}+h_{67} \hat{k}\right|} \\
\Rightarrow \cos \gamma^{\prime}{ }_{L} & =\frac{a_{56} a_{67}+r_{56} r_{67}+h_{56} h_{67}}{\sqrt{a_{56}^{2}+r_{56}^{2}+h_{56}^{2}} \sqrt{a_{67}^{2}+r_{67}^{2}+h_{67}^{2}}} \\
\Rightarrow \gamma^{\prime}{ }_{L} & =\cos ^{-1}\left(\frac{a_{56} a_{67}+r_{56} r_{67}+h_{56} h_{67}}{\sqrt{a_{56}^{2}+r_{56}^{2}+h_{56}^{2}} \sqrt{a_{67}^{2}+r_{67}^{2}+h_{67}^{2}}}\right)
\end{aligned}
$$

Therefore, the inner knee angle or obtuse knee angle for the left leg,

$$
\beta_{L}^{\prime}=180^{\circ}-\gamma^{\prime}{ }_{L}
$$

Similarly, the acute knee angle $\gamma_{R}{ }^{\prime}$ between $\overrightarrow{R_{T k}}$ and $\overrightarrow{R_{S k}}$ for right leg has been determined in Eq. 10.

$$
\Rightarrow \quad \gamma_{R}^{\prime}=\cos ^{-1}\left(\frac{a_{89} a_{910}+r_{89} r_{910}+h_{89} h_{910}}{\sqrt{a_{89}^{2}+r_{89}^{2}+h_{89}^{2}} \sqrt{a_{910}^{2}+r_{910}^{2}+h_{910}^{2}}}\right)
$$

Therefore, the obtuse angle or inner knee angle for right leg would be,

$$
\beta_{R}^{\prime}=180^{\circ}-\gamma_{R}^{\prime}
$$

\section{G. Bland and Altman (B\&A) Plot Analysis}

The proposed IR-UWB prototype and Kinect have been used here to measure the same gait parameter i.e., knee angle with differences found. Subsequently, the outcomes have been compared using B\&A plot analysis [32], [33] based on the quantification of the agreement between two quantitative measurements by studying the mean difference and constructing limits of agreement to assess the comparability between the methods. The statistical limits are calculated using the mean, standard deviation of the differences between the two measurements, and a hypothetical graphical approach to indicate the agreement. The knee angle of participants has been measured through both the proposed and Kinect systems. Let, the measured knee angles from the proposed and Kinect system be $k_{p}$ and $k_{k}$ respectively, mean of knee angle is $m_{k}$, differences between paired knee angle is $d_{k}$, standard deviation of the differences obtained for knee angle is $s_{k}$. The graphical approach is employed to observe the assumptions of normality of differences and other characteristics where, the $x$-axis represents the average of measurements and $y$-axis shows the difference between the two measurements. The two systems would agree when most of the consequences lie within $d_{k} \pm 1.96 s_{k}$ for the measurement of knee angle. More precisely, $95 \%$ of differences must lie within $d_{k} \pm 1.96 s_{k}$ for measuring the knee angle according to Bland Altman analysis. Thus, the null hypothesis states here there is no significant difference between populations (measurements) when using the proposed gait identification model and Kinect for determining knee angles of participants where probability value $p<0.05$ indicates acceptance of null hypothesis and correctness of assumption.

\section{Result ANALysis}

As explained the experiment has been conducted in two environments: anechoic and multipath to investigate robustness, cost effectiveness, and suitability. In addition to this, the preciseness and acceptance of the work for gait characterisation has been supported through B\&A plot analysis in each environment. The results and B\&A plot analysis are presented in the following sections.

\section{A. Result Analysis from Anechoic Chamber}

The comparative analysis of the obtained results from the proposed prototype and Kinect sensor are demonstrated in this section. The processing of IR-UWB data and interpretation has been discussed in Section [II-C, which explains the positions of back-scattered pulses from a human body and defines motion through the IR-UWB. Figure 5 shows one of the twenty normal walking patterns through the IR-UWB response in 3D over an observation period, where Figure $5 \mathrm{a}$ and $5 \mathrm{~b}$ lay out front and side views of walking motion captured through the proposed model.

The $x, y$, and $z$ axis signify gait motion width, distance from radar, and height of movement respectively. The motion appears like the letter ' $W$ ', showing the symmetry of the human body with three areas labeled $P_{1}, P_{2}$, and $P_{3}$. Here, the area $P_{1}$ reflects the hip joint of that person, $P_{1}$ to $P_{2}$ and 

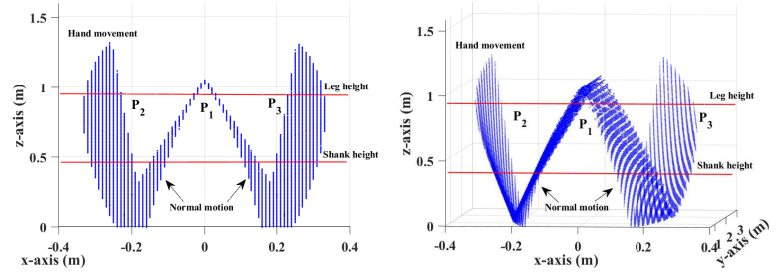

(a) 2D view of IR-UWB 3D (b) Side view of IR-UWB 3D response from a normal walk. response from a normal walk.

Fig. 5: The front and side views of IR-UWB 3D response from a normal walk.

$P_{1}$ to $P_{3}$ denotes the change of position of human body due to gait motion when one leg is lifted off of the ground and another leg is contacted the ground to push forward the body during walking. The person walked back and forth in front of the radar (within the $3 \mathrm{~m}$ testbed) during the observation times creating the distinct areas $\left(P_{1}, P_{2}\right.$, and $\left.P_{3}\right)$ in 3D. Also, the distance between the bottom of $P_{2}$ and $P_{3}$ areas represent the step base width i.e., the perpendicular distance between two steps during gait. In addition, two areas detected above leg height are the hand movements (both right and left). Figure 6a displays the front view of a walking pattern captured through the IR-UWB response and 6b demonstrates the skeletonization of that gait pattern acquired using the Kinect in the anechoic chamber.

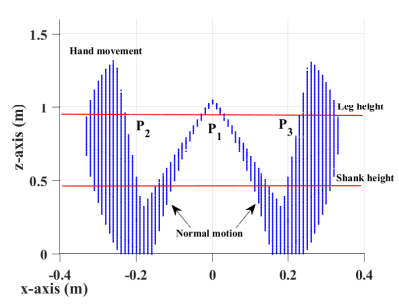

(a) $2 \mathrm{D}$ view of $3 \mathrm{D}$ human motion from IR-UWB.

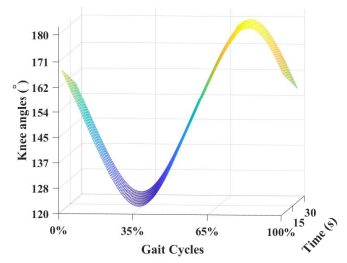

(c) Variation of knee angles determined from proposed model in anechoic chamber.

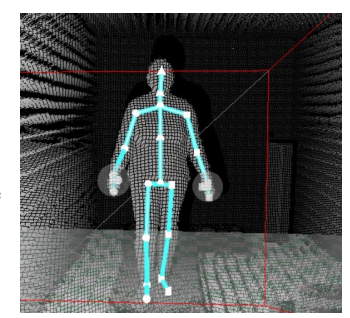
from Kinect.

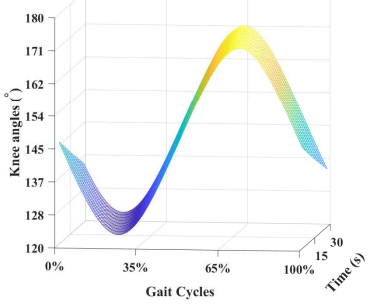

(d) Variation of knee angles determined from skeleton in anechoic chamber
Fig. 6: The human motion and knee angles obtained from the proposed model and Kinect for a person having normal walking pattern.

Figure 6a shows a 3D structure resembling the letter ' $\mathrm{W}$ ' which includes the flexion and extension of the skeletal muscle's (i.e., arm and legs) motion over time. The skeletal muscles move faster than the other body sections implying the transmission of higher energy by the bio-mechanical process that allows UWB radar to capture motion. The extension of lower limbs (left and right) creates a separate motion area, whereas the flexion (right and leg) of the lower limb and upper limbs creates a linear region from the shoulders that explains human motion. The person depicted in $6 \mathrm{a}$ and $6 \mathrm{~b}$ has an actual height of $1.55 \mathrm{~m}$ whereas the estimated height of the shape is $1.35 \mathrm{~m}$. This is because the model has captured all movements by UWB upto the shoulder height from the ground level. The leg length of that participant is $0.95 \mathrm{~m}$ and knee height is $0.45 \mathrm{~m}$ from the ground level have been used to separate each lower limb sections to determine the left and right knee angles. Figure $6 \mathrm{c}$ and $6 \mathrm{~d}$ demonstrates the estimation of knee angles from the proposed study and Kinect respectively using the method of Eq. 4. 6 and Eq. 8, 10. The $x$-axis denotes the single gait cycle (in percentage) of a person by considering two consecutive steps and the process has been repeated for 30 seconds then plotted in $y$-axis and $z$-axis representing the knee angles during the observation time. The outcomes have been detailed here for 30 seconds for each participant. This participant has walked at a speed of $1.33 \mathrm{~m} / \mathrm{s}$ (obtained from Doppler effect) and the knee angles obtained from the proposed prototype vary between approximately $120^{\circ}$ to $178^{\circ}$ whereas, the angles obtained from Kinect results vary between approximately $122^{\circ}$ to $175^{\circ}$. The troughs here represent the angles during flexion and crest signifies the angles at the time of leg extension.

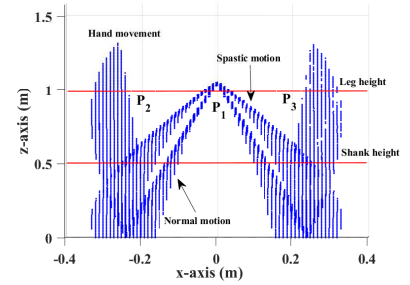

(a) 2D view of IR-UWB 3D response from a abnormal walk

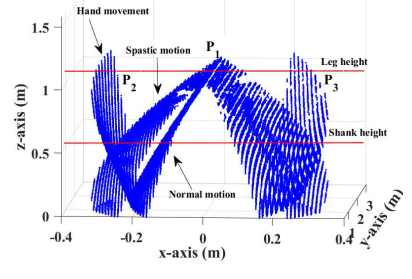

(b) The side view of IR-UWB 3D response from a abnormal walk.
Fig. 7: The front and side views of IR-UWB 3D response from an abnormal walk.

Figure 7] shows one of the abnormal walking patterns through the IR-UWB response in 3D over the same observation period, where Figure $7 \mathrm{a}$ and $7 \mathrm{~b}$ lay out the front and side views of the walking motion captured through the proposed model. The $x, y$, and $z$ axis signify gait motion width, distance from the radar, and height of movement respectively. The motion again appears like the letter ' $\mathrm{W}$ ', shows symmetry of the human body and again there are three areas are labeled with $P_{1}, P_{2}$, and $P_{3}$. But, Figure 7 has differences from Figure 5 Here the abnormality creates two extra regions for the abnormal leg movement (spasticity). Similarly, the area $P_{1}$ reflects the hip joint of that person, $P_{1}$ to $P_{2}$ and $P_{1}$ to $P_{3}$ denotes the change of position of human body due to gait motion when one leg is lifted off of the ground and another leg is contacted to the ground to push the body forward during walking. As before the person walked back and forth in front of radar (within $3 \mathrm{~m}$ of testbed) during observation 
time that creates separate areas $\left(P_{1}, P_{2}\right.$, and $\left.P_{3}\right)$ in $3 \mathrm{D}$ for change of position. Overall the person's movement is effected by their condition in particular one leg is affected by their spasticity, hence the both legs create two separate areas in $P_{2}$ and $P_{3}$. This shows the person needs to stretch and drag one leg more than a regular walker and this motion reflected in the proposed IR-UWB model outcomes. Also, the distance between the bottom of $P_{2}$ and $P_{3}$ areas represents step base width which is also different from normal walk. In addition, two areas detected above leg height are the hand movements (both right and left legs).

Figure 8 displays the gait details of that person through IR-UWB and Kinect. Figure $8 \mathrm{~b}$ displays the motion of the said participant and stiffness of the left leg muscle forces the person to stretch the leg more during walking. Figure 8 a shows that the leg deviates more from the centre of the body during walking, resulting in the unusual knee angle variation between $135^{\circ}$ to $163^{\circ}$ determined from the proposed prototype. The knee angles obtained from Kinect are changes between $139^{\circ}$ to $171^{\circ}$ approximately.

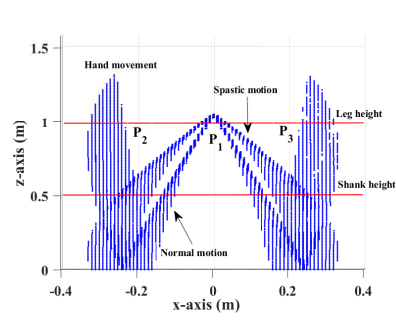

(a) $2 \mathrm{D}$ view of $3 \mathrm{D}$ human motion captured by IR-UWB from spastic gait.
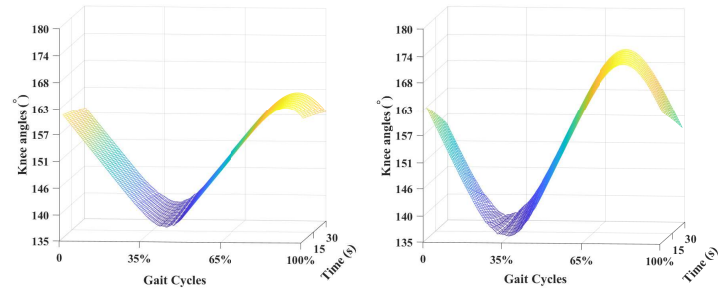

(c) Changes of knee angles (d) Changes of knee andetermined from proposed gles determined from Kinect model for spastic gait. skeleton for spastic gait.

Fig. 8: Human motion and knee angles obtained from proposed model and Kinect respectively for a person with spasticity.

\section{B. B\&A Plot: Results Obtained in Anechoic Chamber}

The results obtained from the proposed model and Kinect system have been corroborated through a B\&A plot analysis. Theoretical details of B\&A plot has been demonstrated in II-G to support the measurement of knee angle utilising the proposed model. Figure 9 shows B\&A plots have been constructed on knee angle measurements from the proposed prototype and Kinect sensor, where Figure 9a demonstrates B\&A plots of knee angle measurements taken from twenty normal gait persons using the proposed prototype and Kinect respectively and Figure $9 \mathrm{~b}$ demonstrates B\&A plots of knee angle measurements taken for four abnormal gait persons from the proposed prototype and Kinect system. The $x$ and $y$ axis represents the mean of the two measurements and difference between two paired measurements respectively. Both, the developed model and Kinect methods imply some degree of error but B\&A plot indicates relationship and agreement between these two methods for gait analysis. Figure 9a shows the bias or mean of difference is -0.847 , signifying the second method here Kinect always produces $0.847^{\circ}$ units more than the proposed model and $95 \%$ differences are within $d_{k} \pm 1.96 s_{k}$ while measuring knee angles. In addition, Figure 9 b displays the bias at -2.242 when measuring abnormal gait patterns, indicating Kinect always delivers $2.242^{\circ}$ units more than the designed prototype for the measurement of knee angles and $95 \%$ differences are within $d_{k} \pm 1.96 s_{k}$ in this context. Thus, both cases suggest the null hypothesis (there is no significant difference between the proposed prototype and Kinect system's magnitude while measuring knee angles) is true and the developed model would be an alternative gait analysis method for consideration.

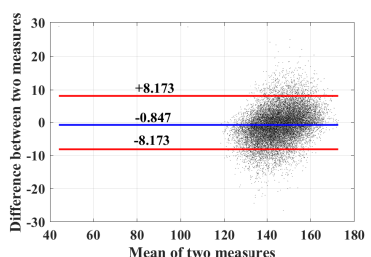

(a) B\&A plot of knee angles from normal gaits.

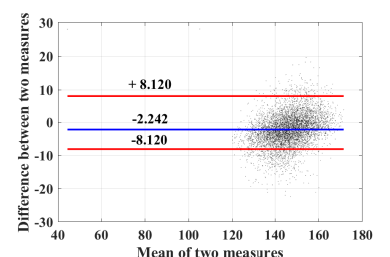

(b) B\&A plot of knee angles from abnormal gaits.
Fig. 9: B\&A plot of obtained knee angles experimented in anechoic chamber.

\section{Result Analysis from Multipath Environment}

Figures 10a and 10b illustrate the 3D motion captured from a person's gait by the proposed prototype and Kinect respectively in the multipath environment (laboratory and corridoor environment). Figures 10c and 10d demonstrate the obtained knee angles from the proposed and Kinect systems respectively. The $x$-axis of Figures $10 \mathrm{c}$ and $10 \mathrm{~d}$ indicate the fractions of gait cycles covered by the knee angles during the observation time where, each gait cycle contains two consecutive steps (stance $\approx 60 \%$ and swing $\approx 40 \%$ phase) with respect to the reference leg (left leg in this case) involved in the walking. The knee angle increases nearly at the time while the reference leg is in the swing phase and decreasing in the stance phase, is shown in Figures $10 \mathrm{c}$ and $10 \mathrm{~d}$. The variation of knee angles has been captured in the same way from the proposed IR-UWB and Kinect system (in Figure $10 \mathrm{c}$ and $10 \mathrm{~d}$. The knee angle varies between $115^{\circ}$ to $163^{\circ}$ and between $122^{\circ}$ to $163^{\circ}$ while using proposed prototype and Kinect system respectively.

\section{B\&A Plot: Results Obtained in Multipath Environment}

Here, the measurements taken in the multipath environment (laboratory environment) has been supported through B\&A 


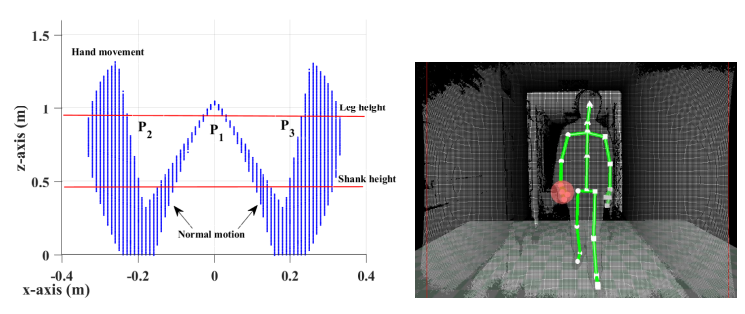

(a) 2D view of 3D human mo- (b) 3D human skeleton tion obtained from IR-UWB in obtained from Kinect in multipath environment. multipath environment.
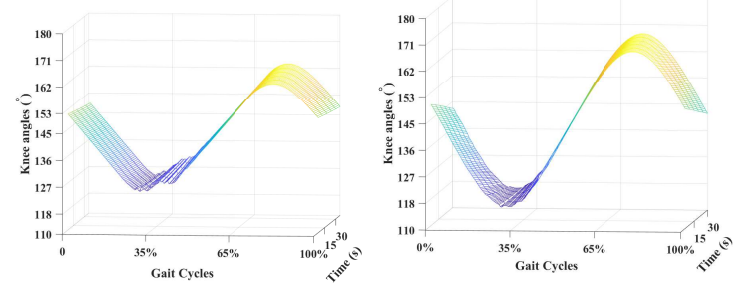
(c) Variation of knee angles (d) Variation of knee andetermined from proposed skeleton in anechoic chammodel in anechoic chamber. ber.

Fig. 10: The human motion and knee angles obtained from proposed model and Kinect respectively for a person having normal walking pattern in multipath environment.

plot analysis same as for anechoic chamber experiment. Figure 11 shows the B\&A plots constructed on knee angle measurements from the developed prototype and Kinect sensor, where Figure 11a demonstrates B\&A plots of knee angle measurements taken for twenty 'normal' gait persons from the designed framework and Kinect with Figure $11 \mathrm{~b}$ demonstrating the B\&A plots of the knee angle measurements taken for four abnormal gait persons via the developed system and Kinect in the normal or multipath environment. The $x$ and $y$ axis represent the mean of two knee angle measurements and the differences between their paired measurements respectively. Figure 11a shows the bias or mean of difference is -0.902 which signifies that Kinect always produces $0.902^{\circ}$ units more than the proposed work to determine knee angles of normal gait persons where $p$ value is $1.0406 \times 10^{-6}$ (i.e., $p<0.05$ ). In addition, Figure $11 \mathrm{~b}$ displays the bias at -2.514 when measuring knee angles of abnormal gait persons indicating Kinect always delivers $2.514^{\circ}$ degree units more than the constructed framework for the measurement of the knee angles, where $p$ is 0.0017 (i.e., $p<0.05$ ). Thus, the null hypothesis have been found to be true in both cases (normal and abnormal gaits) in normal environment, $95 \%$ differences are within $d_{k} \pm 1.96 s_{k}$ and there is no significant difference between the proposed framework and Kinect system's magnitude while measuring knee angles in normal environment. This shows the system has potential suitability for NWS gait analysis.

\section{Discussion \& CONCLUSION}

In this work, for the first time a proposed 3D model of human motion has been generated from non-contact, camera free, IR-UWB sensing by employing trigonometry and vector

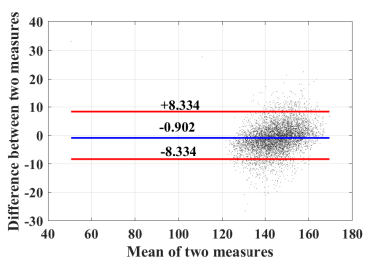

(a) B\&A plot of knee angles from normal gaits.

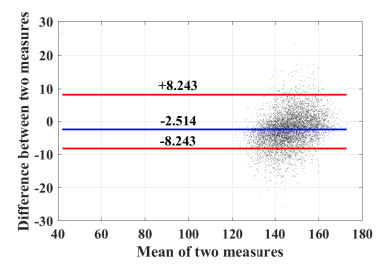

(b) B\&A plot of knee angles from abnormal gaits.
Fig. 11: B\&A plot of obtained knee angles experimented in normal environment.

algebra where, subjective knowledge enabled the study to further characterize human gait. The scope of the current work considered identification of the knee angles only. The implementation execution time is proportional to the square of the scan number (or, number of received pulses). Thus, the study requires quadratic time in real scenario which costs $O\left(n^{2}\right)$ running time. Subsequently, a greater number of participants, including those with conditions such as, propulsive, waddling, steppage, etc. will be considered for future experiments. The Kinect sensor has been employed as a reference system used to evaluate the performance of proposed model. The experiment has been conducted in both an anechoic chamber and 'normal' environment where the proposed prototype and Kinect sensor have an accuracy of approximately $9 \mathrm{~mm}$ and $18 \mathrm{~mm}$ [34] respectively. Furthermore, Kinect suffers from the self-occlusion problem where the one half of the body is occluded by the other each time the participant turns around at the end of test bed. This problem only occurs at the $0^{\circ}$ azimuth beam angle in IR-UWB model, whereas Kinect results are biased in $0^{\circ}, 30^{\circ}, 60^{\circ}$ affecting the Kinect sensor. It has been also reported that the Kinect shows an error greater than $5^{\circ}$ in clinical settings for knee angle measurements [31]. Though, here it has been found that the Kinect delivers $0.047^{\circ}$ (normal walks) and $2.242^{\circ}$ (abnormal walks) more in the anechoic environment, whereas $0.902^{\circ}$ (normal walks) and $2.514^{\circ}$ (abnormal walks) more in the real environment for the knee angle computation than proposed work, explored through B\&A analysis. The difference between the results obtained from the two devices occurs because of the self-occlusion problem and the number of joints detected. The Kinect is more biased in terms of self-occlusion. Also, Kinect only consider points over joints whether the problems may belong to somewhere between the joints, Kinect fails to represent this, whereas the proposed IR-UWB creates a rendered geometric pattern and detects more points all over the body which is capable of detecting abnormalities more precisely. Hence, the obtained experimental result demonstrate the new system's obvious potential, producing equivalent or better results than Kinect sensor. The proposed model is a cutting edge solution to address gait disability and monitoring by non-contact IRUWB technology as a plug and play option e.g., field zones, local home systems or care homes. Further, this study will now be extended by employing supervised machine learning techniques to automatically recognise changes and identify human walking disorders, which involves dedicated sports lab- 
oratory conditions to perform gold standard test comparisons and realise supervised machine learning (SML) algorithms to identify abnormality in gait automatically. This would provide a cutting-edge game changing and widely deployable solution in health and medical perspective to assist in clinical and pathological gait diagnosis.

\section{REFERENCES}

[1] Michael W Whittle. Gait analysis: an introduction. ButterworthHeinemann, 2014.

[2] Jenna Fletcher. What is abnormal gait? https://www.medicalnewstoday. com/articles/320481.php. Sun 31 December 2017. [Online; accessed 10-03-2019].

[3] Alvaro Muro-De-La-Herran, Begonya Garcia-Zapirain, and Amaia Mendez-Zorrilla. Gait analysis methods: An overview of wearable and non-wearable systems, highlighting clinical applications. Sensors, 14(2):3362-3394, 2014.

[4] U Chris Ugbolue, Enrica Papi, Konstantinos T Kaliarntas, Andrew Kerr, Leo Earl, Valerie M Pomeroy, and Philip J Rowe. The evaluation of an inexpensive, $2 \mathrm{~d}$, video based gait assessment system for clinical use. Gait \& posture, 38(3):483-489, 2013.

[5] Alexandra Pfister, Alexandre M West, Shaw Bronner, and Jack Adam Noah. Comparative abilities of microsoft kinect and vicon $3 \mathrm{~d}$ motion capture for gait analysis. Journal of medical engineering \& technology, 38(5):274-280, 2014.

[6] S. Corazza, E. Gambaretto, L. Mundermann, and T. P. Andriacchi. Automatic generation of a subject-specific model for accurate markerless motion capture and biomechanical applications. IEEE Transactions on Biomedical Engineering, 57(4):806-812, April 2010.

[7] Dong-Hwan Lim, Wan-Soo Kim, Ho-Jun Kim, and Chang-Soo Han. Development of real-time gait phase detection system for a lower extremity exoskeleton robot. International Journal of Precision Engineering and Manufacturing, 18(5):681-687, 2017.

[8] J. C. Y. Lai, Y. Xu, E. Gunawan, E. C. Chua, A. Maskooki, Y. L. Guan, K. Low, C. B. Soh, and C. Poh. Wireless sensing of human respiratory parameters by low-power ultrawideband impulse radio radar. IEEE Transactions on Instrumentation and Measurement, 60(3):928938, 2011.

[9] E. Piuzzi, P. D'Atanasio, S. Pisa, E. Pittella, and A. Zambotti. Complex radar cross section measurements of the human body for breath-activity monitoring applications. IEEE Transactions on Instrumentation and Measurement, 64(8):2247-2258, 2015.

[10] Y. S. Koo, L. Ren, Y. Wang, and A. E. Fathy. Uwb microdoppler radar for human gait analysis, tracking more than one person, and vital sign detection of moving persons. In 2013 IEEE MTT-S International Microwave Symposium Digest (MTT), pages 1-4, June 2013.

[11] A. Seifert, M. G. Amin, and A. M. Zoubir. New analysis of radar micro-doppler gait signatures for rehabilitation and assisted living. In 2017 IEEE International Conference on Acoustics, Speech and Signal Processing (ICASSP), pages 4004-4008, March 2017.

[12] Ghassem Mokhtari, Qing Zhang, Chad Hargrave, and Jonathon C Ralston. Non-wearable uwb sensor for human identification in smart home. IEEE Sensors Journal, 17(11):3332-3340, 2017.

[13] Sasanka Potluri, Srinivas Ravuri, Christian Diedrich, and Lutz Schega Deep learning based gait abnormality detection using wearable sensor system. In 2019 41st Annual International Conference of the IEEE Engineering in Medicine and Biology Society $(E M B C)$, pages 36133619. IEEE, 2019.

[14] Jing Gao, Peishang Gu, Qing Ren, Jinde Zhang, and Xin Song. Abnormal gait recognition algorithm based on lstm-cnn fusion network. IEEE Access, 7:163180-163190, 2019.

[15] L. Ren, N. Tran, F. Foroughian, K. Naishadham, J. E. Piou, O. Kilic, and A. E. Fathy. Short-time state-space method for micro-doppler identification of walking subject using uwb impulse doppler radar. IEEE Transactions on Microwave Theory and Techniques, 66(7):3521-3534, July 2018.

[16] Irena Orović, Srdjan Stanković, and Moeness Amin. A new approach for classification of human gait based on time-frequency feature representations. Signal Processing, 91(6):1448-1456, 2011.

[17] S. Zihajehzadeh, P. K. Yoon, B. Kang, and E. J. Park. Uwb-aided inertial motion capture for lower body 3-d dynamic activity and trajectory tracking. IEEE Transactions on Instrumentation and Measurement, 64(12):3577-3587, 2015.
[18] Salim Ghoussayni, Christopher Stevens, Sally Durham, and David Ewins. Assessment and validation of a simple automated method for the detection of gait events and intervals. Gait \& Posture, 20(3):266-272, 2004.

[19] Dustin A Bruening and Sarah Trager Ridge. Automated event detection algorithms in pathological gait. Gait \& posture, 39(1):472-477, 2014.

[20] Barry R. Greene, Denise McGrath, Ross O'Neill, Karol J. O’Donovan, Adrian Burns, and Brian Caulfield. An adaptive gyroscope-based algorithm for temporal gait analysis. Medical \& Biological Engineering \& Computing, 48(12):1251-1260, Dec 2010.

[21] F. Bugané, M.G. Benedetti, G. Casadio, S. Attala, F. Biagi, M. Manca, and A. Leardini. Estimation of spatial-temporal gait parameters in level walking based on a single accelerometer: Validation on normal subjects by standard gait analysis. Computer Methods and Programs in Biomedicine, 108(1):129 - 137, 2012.

[22] CONTEMPLAS. Templo clinical gait analysis. https://www.contemplas. com/clinical-gait-analysis.aspx Online accessed: December, 2017.

[23] Tekscan. Pressure mapping, force measurement \& tactile sensors. https://www.tekscan.com/gait-analysis-systems Online accessed: January, 2018.

[24] Soumya Prakash Rana, Maitreyee Dey, Mohammad Ghavami, and Sandra Dudley. ITERATOR: A 3d gait identification from ir-uwb technology. In 2019 41st Annual International Conference of the IEEE Engineering in Medicine and Biology Society (EMBC), pages 782-787. IEEE, 2019

[25] Soumya Prakash Rana et al. UWB localization employing supervised learning method. In IEEE 17th International Conference on Ubiquitous Wireless Broadband (ICUWB), Salamanca, Spain, 2017.

[26] Soumya Rana et al. Remote vital sign recognition through machine learning augmented UWB. In IET 12th European Conference on Antennas and Propagation (EuCAP), London, United Kingdom, April, 2018.

[27] Soumya Prakash Rana, Maitreyee Dey, Mohammad Ghavami, and Sandra Dudley. Signature inspired home environments monitoring system using ir-uwb technology. Sensors, 19(2):385, 2019.

[28] S. P. Rana, M. Dey, M. Ghavami, and S. Dudley. Non-contact human gait identification through ir-uwb edge-based monitoring sensor. IEEE Sensors Journal, 19(20):9282-9293, 2019.

[29] Federal Communications Commission. In the matter of revision of part 15 of the commission's rules regarding ultra-wideband transmission systems. First Report And Order, ET Docket 98-153, 2002.

[30] Baobin Li, Changye Zhu, Shun Li, and Tingshao Zhu. Identifying emotions from non-contact gaits information based on microsoft kinects. IEEE Transactions on Affective Computing, 9(4):585-591, 2016.

[31] Alexandra Pfister, Alexandre M West, Shaw Bronner, and Jack Adam Noah. Comparative abilities of microsoft kinect and vicon $3 \mathrm{~d}$ motion capture for gait analysis. Journal of medical engineering \& technology, 38(5):274-280, 2014

[32] J Martin Bland and DouglasG Altman. Statistical methods for assessing agreement between two methods of clinical measurement. The lancet, 327(8476):307-310, 1986.

[33] Davide Giavarina. Understanding bland altman analysis. Biochemia medica: Biochemia medica, 25(2):141-151, 2015.

[34] Oliver Wasenmüller and Didier Stricker. Comparison of kinect v1 and v2 depth images in terms of accuracy and precision. In Asian Conference on Computer Vision, pages 34-45. Springer, 2016. 